\title{
Co-Expression and Co-Localization of Cartilage Glycoproteins CHI3L1 and Lubricin in Osteoarthritic Cartilage: Morphological, Immunohistochemical and Gene Expression Profiles
}

\author{
Marta Anna Szychlinska ${ }^{1}$, Francesca Maria Trovato ${ }^{2}$, Michelino Di Rosa ${ }^{3}$, Lucia Malaguarnera ${ }^{3}$, \\ Lidia Puzzo ${ }^{4}$, Rosy Leonardi ${ }^{5}$, Paola Castrogiovanni ${ }^{1}$ and Giuseppe Musumeci ${ }^{1, *}$ \\ 1 Department of Biomedical and Biotechnological Sciences, Human Anatomy and Histology Section, \\ School of Medicine, University of Catania, Via S. Sofia 87, 95123 Catania, Italy; \\ mszychlinska@unict.it (M.A.S.); pacastro@unict.it (P.C.) \\ 2 Departments of Clinical and Experimental Medicine, Internal Medicine Division, School of Medicine, \\ University of Catania, 95123 Catania, Italy; trovatofrancesca@gmail.com \\ 3 Department of Biomedical and Biotechnological Sciences, Pathology Section, School of Medicine, \\ University of Catania, 95125 Catania, Italy; mdirosa@unict.it (M.D.R.): lucmal@unict.it (L.M.) \\ 4 Department of Medical and Surgical Sciences and Advanced Technologies, Anatomic Pathology Section, \\ School of Medicine, University of Catania, 95123 Catania, Italy; lipuzzo@unict.it \\ 5 Department of Medical and Surgical Science, Section of Dentistry, University of Catania, 95123 Catania, Italy; \\ rleonard@unict.it \\ * Correspondence: g.musumeci@unict.it; Tel.: +39-095-378-2043; Fax: +39-095-378-2034
}

Academic Editor: Charles J. Malemud

Received: 12 December 2015; Accepted: 22 February 2016; Published: 11 March 2016

\begin{abstract}
Osteoarthritis is the most common human arthritis characterized by degeneration of articular cartilage. Several studies reported that levels of human cartilage glycoprotein chitinase 3-like-1 (CHI3L1) are known as a potential marker for the activation of chondrocytes and the progression of Osteoarthritis (OA), whereas lubricin appears to be chondroprotective. The aim of this study was to investigate the co-expression and co-localization of CHI3L1 and lubricin in normal and osteoarthritic rat articular cartilage to correlate their modified expression to a specific grade of OA. Samples of normal and osteoarthritic rat articular cartilage were analyzed by the Kellgren-Lawrence OA severity scores, the Kraus' modified Mankin score and the Histopathology Osteoarthritis Research Society International (OARSI) system for histomorphometric evaluations, and through CHI3L1 and lubricin gene expression, immunohistochemistry and double immuno-staining analysis. The immunoexpression and the mRNA levels of lubricin increased in normal cartilage and decreased in OA cartilage (normal vs. OA, $p<0.01$ ). By contrast, the immunoexpression and the mRNA levels of CHI3L1 increased in OA cartilage and decreased in normal cartilage (normal vs. OA, $p<0.01$ ). Our findings are consistent with reports suggesting that these two glycoproteins are functionally associated with the development of OA and in particular with grade $2 / 3$ of OA, suggesting that in the future they could be helpful to stage the severity and progression of the disease.
\end{abstract}

Keywords: Lubricin; CHI3L1; Osteoarthritis; anterior cruciate ligament transection (ACLT); Immunohistochemistry; mRNA

\section{Introduction}

Osteoarthritis (OA) is the most common human arthritis characterized by deterioration and loss of articular cartilage [1]. OA is the most prevalent joint condition resulting in physical disability resulting in a high economic burden largely attributable to the effects of disability, co-morbid disease, and the 
expense of treatment [2]. The main risk factors involved in the pathogenesis of $\mathrm{OA}$ are genetics, aging, obesity, injury and biomechanical stress [3]. This condition is associated with progressive hyaline articular cartilage loss, low-grade synovitis and alterations in subchondral bone and periarticular tissues [4].

The causes behind OA development and progression continue to remain largely undefined and understanding the molecular pathogenesis of the disease remains a priority. Recent studies have shown that two glycoproteins may be particularly relevant to OA pathogenesis. The human cartilage glycoprotein chitinase 3-like-1 (CHI3L1) is associated with mediators of inflammation [5-7] and cartilage damage involved in the pathogenesis of OA [8]. We have earlier reported an increased expression of this protein in the OA rat model when compared to the control group [9]. Its production has been correlated to joint inflammation and it was suggested that its over-expression could be involved in remodelling and degradation of cartilage in OA joints [9].

Another fundamental aspect of the OA pathophysiological process is represented by the reduced boundary-lubricating ability of synovial fluid [10]. The latter is associated with the reduction of lubricin, one of the major joint lubricants, in both acute and chronic conditions [11]. In this regard lubricin is a glycoprotein that has received considerable attention such as chondroprotective molecule [10-13]. The association between cartilage, boundary lubrication and evident changes in cartilage tissue after injury has not yet been clearly understood, but considerable evidence from the literature indicates that it may predispose the articular cartilage to degenerate and develop OA [12,14]. Hyper expression of lubricin in transgenic mice has been shown to reduce the severity of both age-related OA as well as in a cruciate ligament transection model of OA [15]. The aim of the present study was to investigate, for the first time, the co-expression and co-localization of CHI3L1 and lubricin in normal and osteoarthritic rat articular cartilage from femoral condyles after anterior cruciate ligament transection (ACLT), morphologically by both immunohistochemistry and double immuno-staining to correlate their modified expression to a specific grade of OA. The purpose of this study was to discover potential roles for both glycoproteins in OA pathophysiology and possibly to improve knowledge in this field in order to find new treatments for inflammatory joint diseases. To strengthen our morphological results we also performed gene expression analyses for both glycoproteins. The experimental model adopted in this study was the induction of a moderate OA in rat by ACLT. This model exhibits a number of characteristics similar to human post-traumatic OA and is widely used in this field of research [9]. In order to evaluate the experimental induction of OA, we performed the radiographic Kellgren-Lawrence OA severity scores, by photographical examination and X-ray microtomography imaging, and the histomorphometric evaluation, by the macroscopic Kraus' modified Mankin score and the microscopic histopathology Osteoarthritis Research Society International (OARSI) system.

\section{Results}

\subsection{Radiographic Analysis}

In agreement with the Kellgren and Lawrence classification, rats from control and sham groups (without ACLT) showed intact and normal cartilage structure without signs of cartilage degeneration (Kellgren-Lawrence score, Grade 0), while in the OA group (with ACLT) animals showed moderate OA of the knee (Kellgren-Lawrence score, Grade 2) as shown in Figure 1A. 

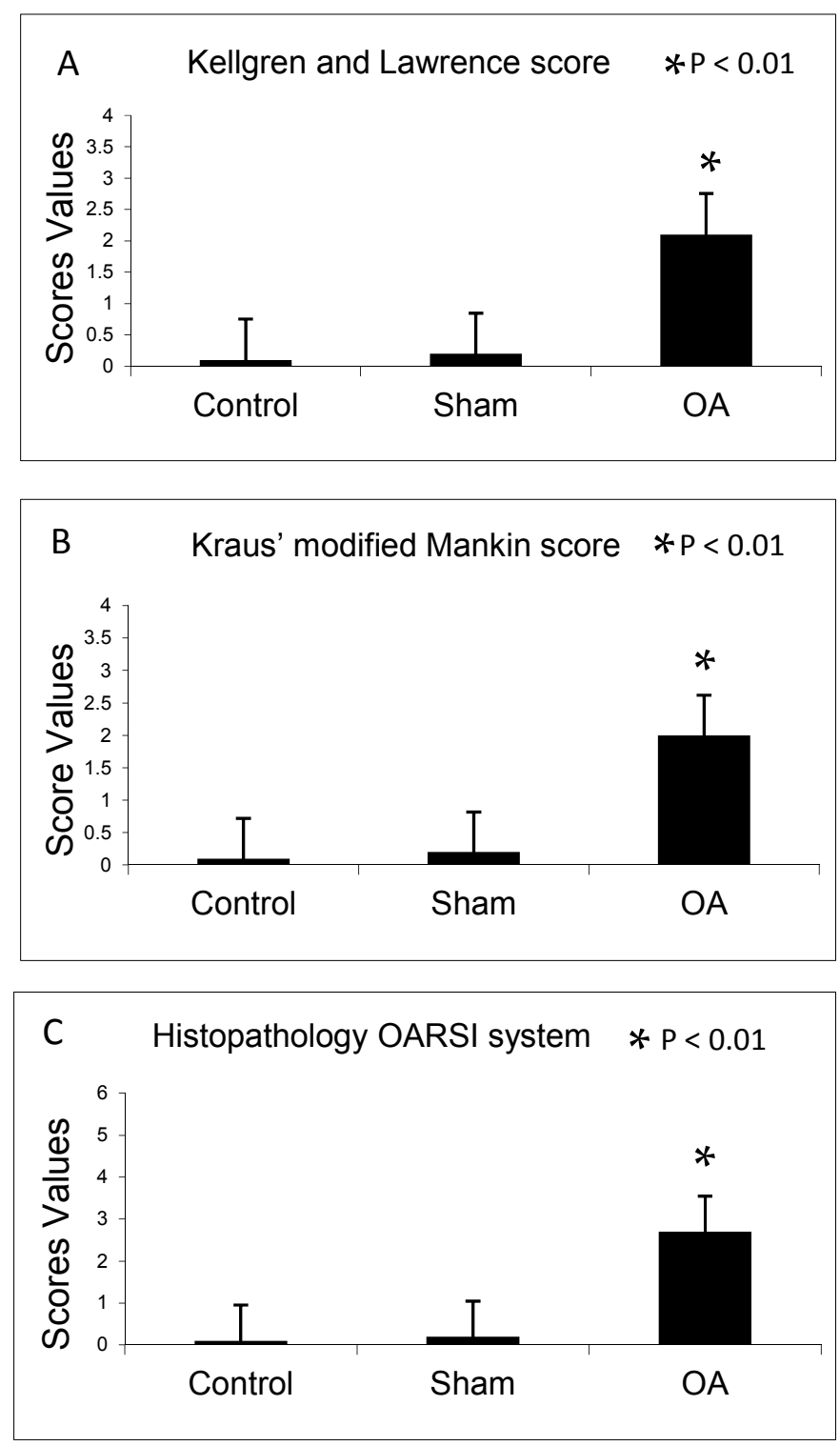

Figure 1. (A) Kellgren and Lawrence score among groups; (B) Kraus' modified Mankin score among groups; and (C) Histopathology Osteoarthritis Research Society International (OARSI) system among groups. Results are presented as the mean \pm SEM. Analysis Of Variance (ANOVA), was used to evaluate the significance of the results. ${ }^{*} p<0.01$, when compared to the control groups. OA: Osteoarthritis.

\subsection{Histomorphometric Analyses}

The histomorphometric parameters made in both control and sham groups, confirmed the presence of intact and normal cartilage structure without signs of cartilage degeneration indeed both Kraus' modified Mankin and Histopathology OARSI system scores were 0. In the OA group, instead, cartilage showed more serious pathological changes, consistent with moderate OA with a Kraus' modified Mankin score of 2 and Histopathology OARSI system score between 2 and 3. Thus, the OA group articular cartilage showed signs of degeneration significantly different from the control groups, as confirmed by Kraus' modified Mankin score (Figures 1B and 2), and histopathology OARSI system (Figures $1 \mathrm{C}$ and 2). 


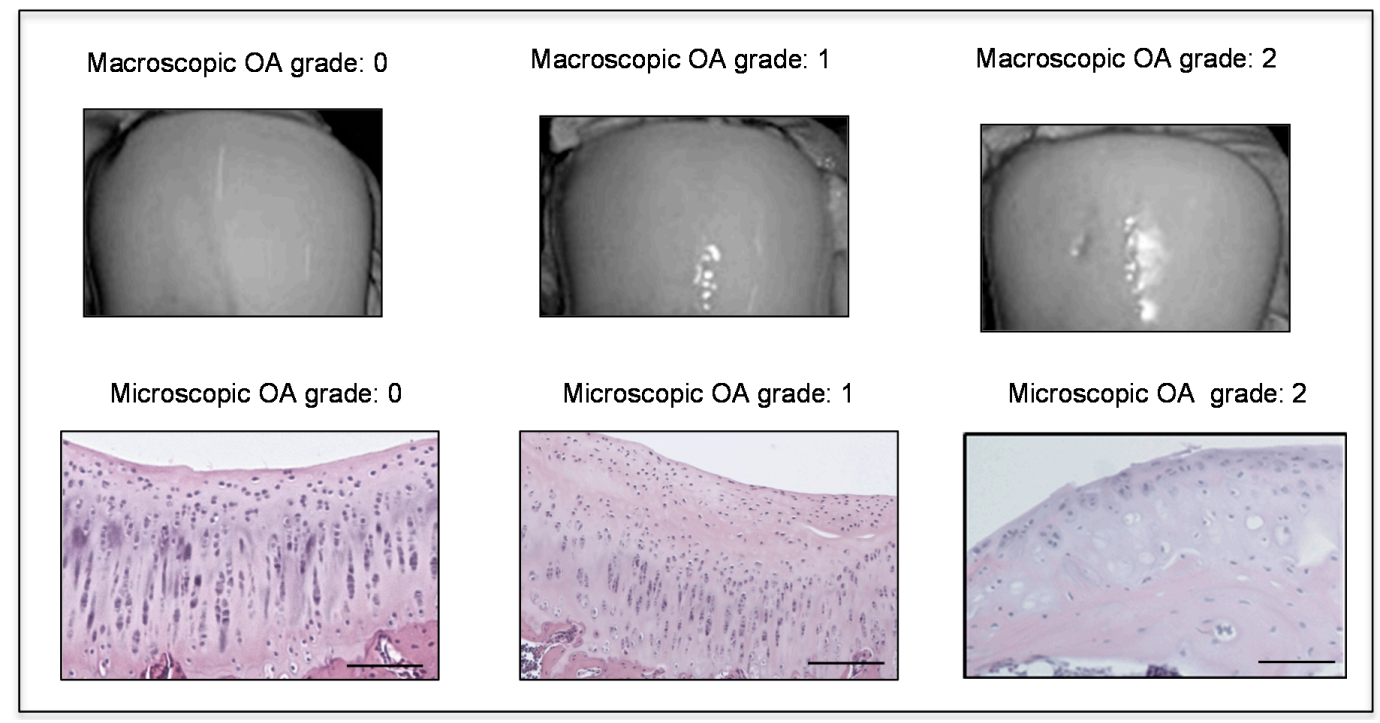

Figure 2. Macroscopic and microscopic articular cartilage degeneration between OA grade 0 to OA grade 2 according to macroscopic Kraus' modified Mankin score and microscopic histopathology OARSI system, Magnifications $\times 20$, scale bars $100 \mu \mathrm{m}$.

\subsection{Immunohistochemistry (IHC) Observations}

CHI3L1 and lubricin were assessed by immunohistochemical staining in cartilage of all groups. Different patterns of immunopositive cells in the sets of specimens were observed (Table 1).

Table 1. Evaluation of lubricin and chitinase 3-like-1 (CHI3L1) immunostaining. Intensity immunostaining (IS); Percentage of immunopositive cells expressed by Extent Score (ES): $<5 \%(0)$; $5 \%-30 \%(+) ; 31 \%-50 \%(++) ; 51 \%-75 \%(+++)$, and $>75 \%(++++)$. ACLT: anterior cruciate ligament transection; OA: Osteoarthritis.

\begin{tabular}{ccc}
\hline Groups & Lubricin & CHI3L1 \\
\hline Control rats without ACLT & $\begin{array}{c}\text { Very strong immunostaining } \\
(\mathrm{ES}=+++; \text { IS }=4)\end{array}$ & $\begin{array}{c}\text { Weak } / \text { absent immunostaining } \\
(\mathrm{ES}=+; \text { IS }=1)\end{array}$ \\
\hline Sham operated control rats & $\begin{array}{c}\text { Very strong immunostaining } \\
(\mathrm{ES}=+++; \text { IS }=4)\end{array}$ & $\begin{array}{c}\text { Weak } / \text { absent immunostaining } \\
(\mathrm{ES}=+; \text { IS }=1)\end{array}$ \\
\hline Experimental rats with ACLT $(\mathrm{OA})$ & $\begin{array}{c}\text { Weak } / \text { absent immunostaining } \\
(\mathrm{ES}=+; \mathrm{IS}=1)\end{array}$ & $\begin{array}{c}\text { Strong immunostaining } \\
(\mathrm{ES}=+++; \text { IS }=3)\end{array}$ \\
\hline
\end{tabular}

CHI3L1 overexpression was found in chondrocytes from the OA group mainly in the middle and deep zone of the cartilage rather than the superficial zone, while it was weakly expressed in cartilage from superficial, middle and deep zone of control and sham groups (Figure 3). CHI3L1 immunolabeling was weak/absent (ES = +; IS =1) in control and sham groups (Figure 3A,B) and was strong (ES = +++; IS =3) in cartilage from OA group (Figure 3C). The negative control treated with PBS without the primary antibody (CHI3L1) did not show immunostaining $(E S=0 ;$ IS $=0)$ as shown in Figure 3D. The percentage of CHI3L1-positive cells was identified among groups ( $p<0.01$ vs. others) as shown in Figure 3E. Interobserver agreement, measured as Cohen's k coefficient, was 0.88. 

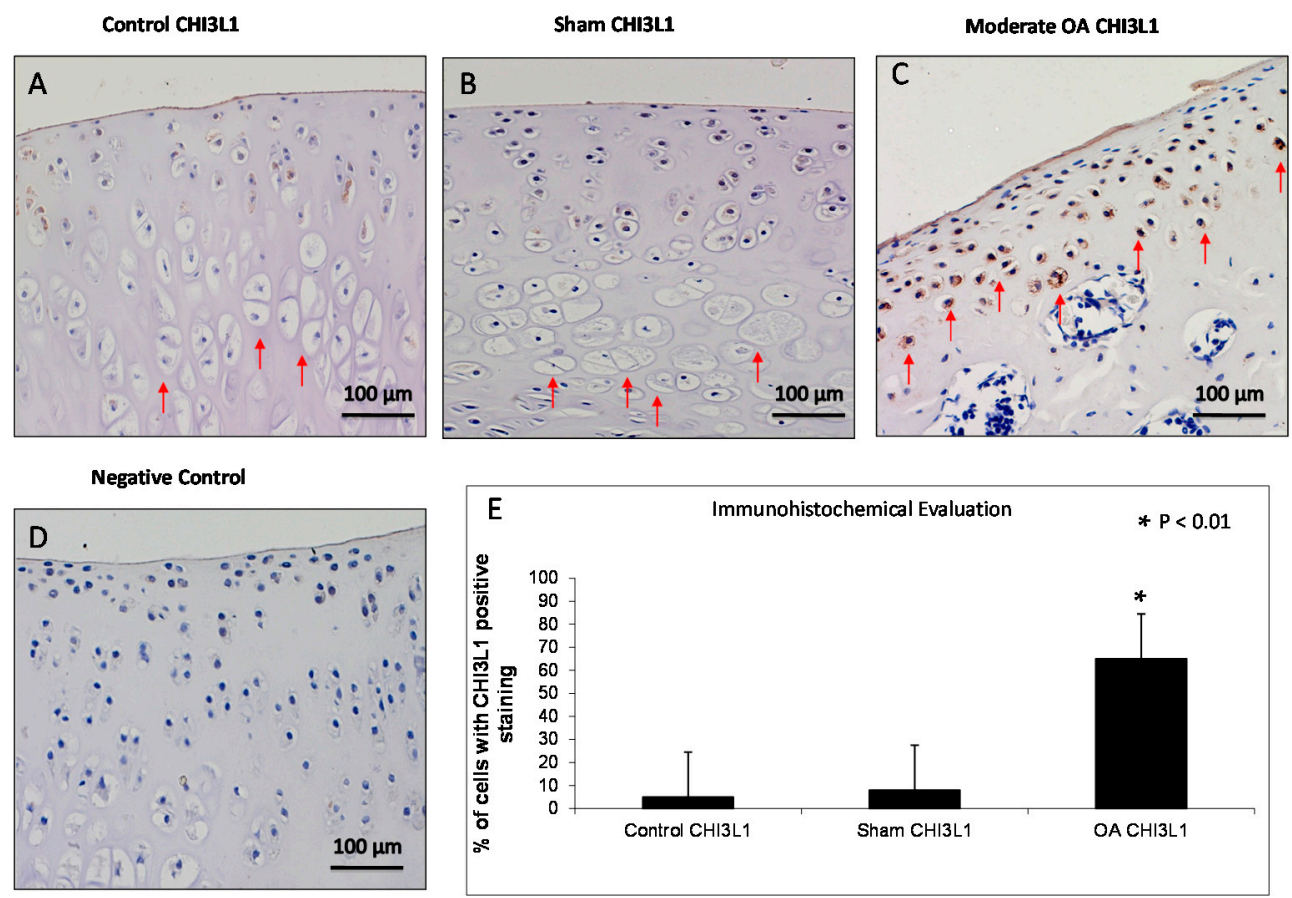

Figure 3. Evaluation of chitinase 3-like-1 (CHI3L1) immunostaining. (A,B) CHI3L1 immunohistochemistry specimen from control (A) and sham (B) rat femoral articular cartilage (without anterior cruciate ligament transection (ACLT)) showed a weak/absent (Extent Score (ES) = +; Intensity immunostaining $(\mathrm{IS})=1$ ) immunostaining in cartilage superficial, middle and deep zone in which hypertrophic chondrocytes are evident (red arrows); (C) CHI3L1 immunohistochemistry specimen from moderate OA rat femoral articular cartilage (with ACLT) exhibited a strong (ES = +++; IS = 3) immunostaining in middle and deep cartilage zone (red arrows) and a reduction of cartilage thickness of the superficial and the middle zones is evident and in the deep zone the chondrocytes are not hypertrophic and are not arranged in columns; (D) The negative control treated with PBS without the primary antibody (CHI3L1) did not show immunostaining $(E S=0$; IS = 0). (A-D) Magnifications $\times 20$; Scale bars: $100 \mu \mathrm{m}$; (E) Immunohistochemical evaluation graph: percentage of CHI3L1 positive cells out of the total number of cells counted in control groups and in OA group. Results are presented as the mean \pm SEM. ANOVA was used to evaluate the significance of the results. ${ }^{*} p<0.01$, when compared to the control groups.

Moderate OA cartilage structural variations included a reduction of cartilage thickness of the superficial and the middle zones, clear deep fissures in the articular surface, and reduction of cells from the superficial, intermediate and deep zone, where chondrocytes are not arranged in columns. The tidemark is not intact in all its extension and the subchondral bone shows fibrillation.

In control and in sham groups lubricin overexpression was found mainly in chondrocytes from the superficial and middle zone of the cartilage rather than the deep zone, while it was weakly expressed in cartilage from superficial, middle and deep zone of osteoarthritic cartilage (Figure 4). Lubricin immunolabeling was very strong $(E S=+++$ IS $=4$ ) in the control and in the sham groups (Figure $4 \mathrm{~A}, \mathrm{~B})$ and was weak/absent $(\mathrm{ES}=+; \mathrm{IS}=1$ ) in cartilage from the OA group as shown in Figure 4C. The negative control treated with PBS without the primary antibody (lubricin) did not show immunostaining ( $E S=0$; IS $=0$ ) as shown in Figure 4D. The percentage of lubricin-positive cells was observed among groups $(p<0.01$ vs. others) as shown in Figure 4E. Interobserver agreement, measured as Cohen's $\mathrm{k}$ coefficient, was 0.92 . 

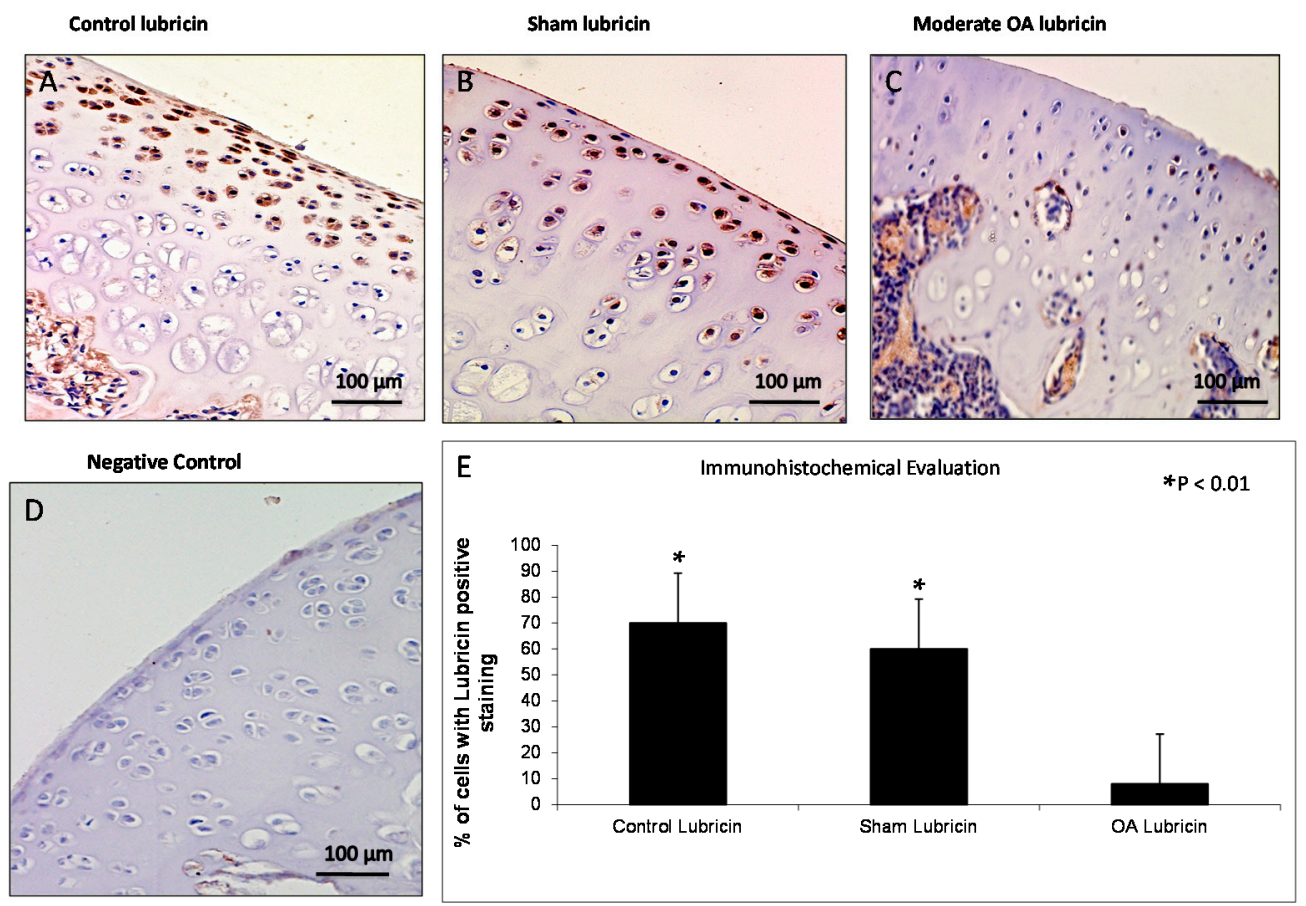

Figure 4. Evaluation of lubricin immunostaining. (A,B) Lubricin immunohistochemistry specimen from control (A) and sham (B) cartilage (without ACLT) showed a very strong (ES = +++; IS = 4) immunostaining in chondrocytes from superficial and middle zone of rat femoral articular cartilage; (C) Lubricin immunohistochemistry specimen from moderate OA cartilage (with ACLT) showed a weak/absent (ES = +; IS = 1) immunostaining in chondrocytes from rat femoral articular cartilage (superficial, middle and deep zone); (D) The negative control treated with PBS without the primary antibody (lubricin) did not show immunostaining $(E S=0$; IS $=0$ ). (A-D) Magnifications $\times 20$; Scale bars: $100 \mu \mathrm{m}$; (E) Immunohistochemical evaluation graph: percentage of lubricin positive cells out of the total number of cells counted in control groups and in the OA group. Results are presented as the mean \pm SEM. ANOVA was used to evaluate the significance of the results. ${ }^{*} p<0.01$, when compared to the control groups.

\subsection{Double Immunostaining Observations}

Double staining was performed with the specific antibodies against lubricin (red) and CHI3L1 (brown) to investigate their expression and to assess their distribution in normal and osteoarthritic articular cartilage tissue. This double stain technique allows us to identify the localization of these two studied proteins. With this technique, we have strengthened and confirmed our previous results, as can be seen from the data presented subsequently. In the control (Figure 5A) and in the sham (Figure 5C) groups, lubricin immunolabeling was strong (ES $=+++$; IS $=3$, red staining), instead the expression of CHI3L1 was weak/absent (ES = +; IS = 1, brown staining). The percentage of lubricin and CHI3L1-positive cells was observed in the control group (lubricin vs. CHI3L1, $p<0.01$ ) as shown in Figure 5B. Interobserver agreement, measured as Cohen's k coefficient, was 0.90. The percentage of lubricin and CHI3L1-positive cells was observed in the sham group (lubricin vs. CHI3L1, $p<0.01$ ) as shown in Figure 5D. Interobserver agreement, measured as Cohen's $\mathrm{k}$ coefficient, was 0.86. In the OA group (Figure 5E) lubricin immunolabeling was weak/absent (ES = +; IS = 1, red staining), instead the expression of CHI3L1 was strong (ES $=+++$; IS $=3$, brown staining). The expression of CHI3L1 increases with the intensification of the cartilage damage. The percentage of lubricin and CHI3L1-positive cells was observed in moderate OA (lubricin vs. CHI3L1, $p<0.01$ ) as shown in Figure 5F. Interobserver agreement, measured as Cohen's k coefficient, was 0.96. Lubricin/CHI3L1 ratio was quantified in control and OA groups. The ratio clearly demonstrated the increased expression of lubricin in control cartilage and the increased expression of CHI3L1 in OA cartilage, conversely the 
decreased expression of lubricin was found in OA cartilage and the decreased expression of CHI3L1 was found in the control cartilage.

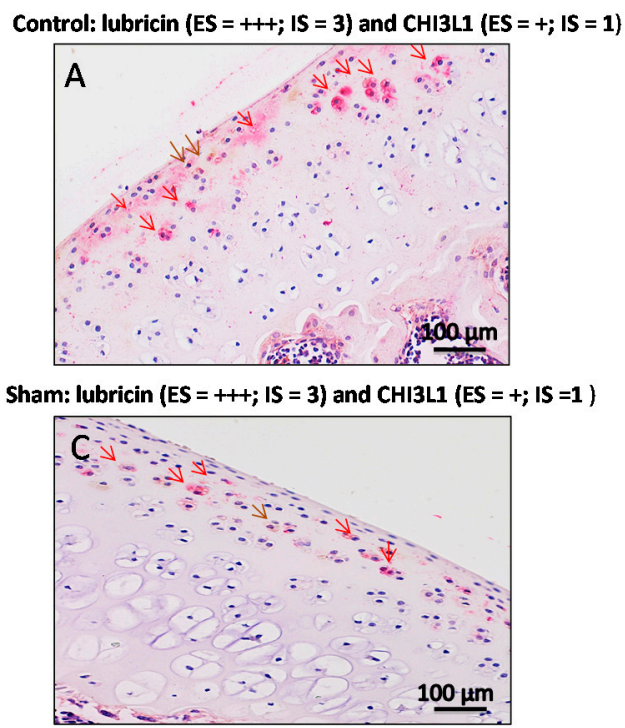

Moderate OA: lubricin (ES = 1; IS = 1) and CHI3L1 (ES =+++; IS = 3)

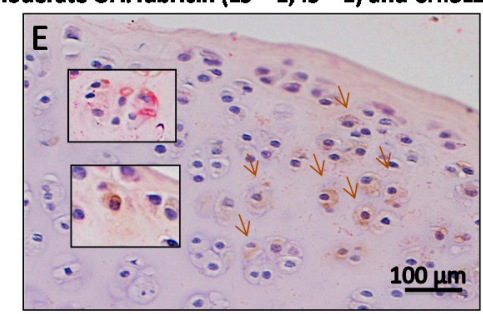

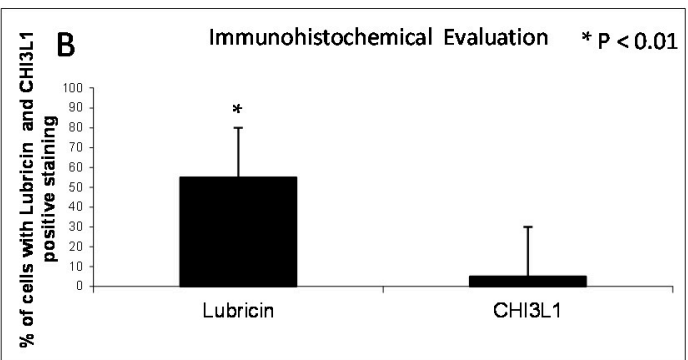
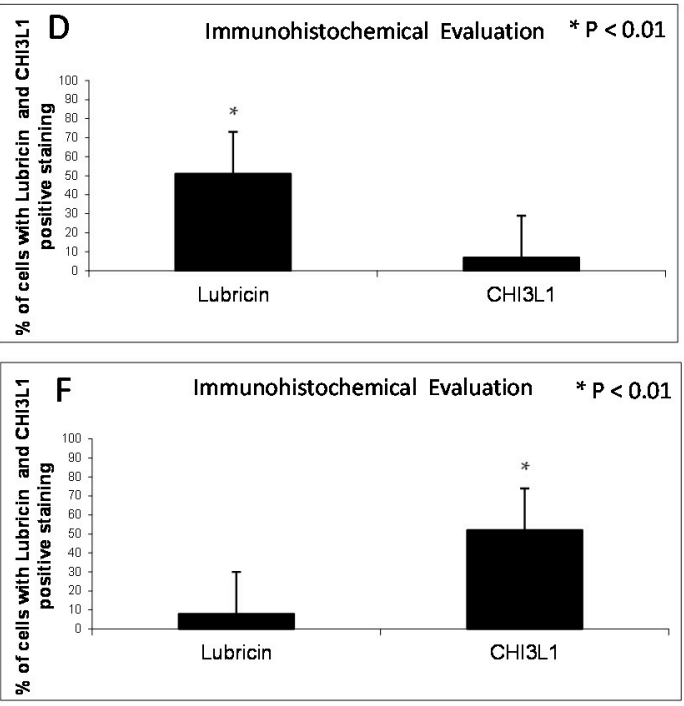

Figure 5. Evaluation of lubricin and CHI3L1 double staining in control and in moderate OA cartilage. $(A, C)$ In control and in sham groups lubricin immunolabeling was strong $(E S=+++; \mathrm{IS}=3$, red staining, red arrows), instead the expression of $\mathrm{CHI} 3 \mathrm{~L} 1$ was weak/absent $(\mathrm{ES}=+; \mathrm{IS}=1$, brown staining, brown arrow); (E) In moderate OA, lubricin immunolabeling was weak/absent $(E S=+; \mathrm{IS}=1$, red staining, red arrows), instead the expression of $\mathrm{CHI} 3 \mathrm{~L} 1$ was strong (ES $=+++; \mathrm{IS}=3$, brown staining, brown arrows); (A,C,E) Magnifications $\times 20$; Scale bars: $100 \mu \mathrm{m}$; inserts: magnifications $\times 40$; Scale bars: $50 \mu \mathrm{m}$; (B) Immunohistochemical evaluation graph: percentage of lubricin and CHI3L1-positive cells out of the total number of cells counted in the control group; (D) Immunohistochemical evaluation graph: percentage of lubricin and CHI3L1-positive cells out of the total number of cells counted in the sham group; (F) Immunohistochemical evaluation graph: percentage of lubricin and CHI3L1-positive cells out of the total number of cells counted in the moderate OA group. Results are presented as the mean \pm SEM. Student's $t$ test, was used to evaluate the significance of the results. ${ }^{*} p<0.01$, when compared lubricin vs. CHI3L1.

\subsection{Chitinase 3-Like-1 (CHI3L1) and Lubricin mRNA Expression in Osteoarthritic Rat Cartilage Model}

The PCR analysis demonstrated that the CHI3L1 mRNA expression significantly increased in OA cartilage (fold 3.2, $p<0.001)$ compared to the sham and control groups $(p<0.001)$ as shown in Figure 6A. Opposite results were obtained for lubricin, in fact lubricin mRNA expression was significantly reduced in OA cartilage (fold $0.47, p<0.001$ ) compared to the sham and control groups $(p<0.001)$ as shown in Figure 6B. Non-significant results were obtained comparing the sham and the control. These data unequivocally confirm the results obtained by immunohistochemistry, supporting the possible relationship between the opposite CHI3L1- and lubricin-expression and the progression of $\mathrm{OA}$, in particular grade $2 / 3$ of OA. 
A)

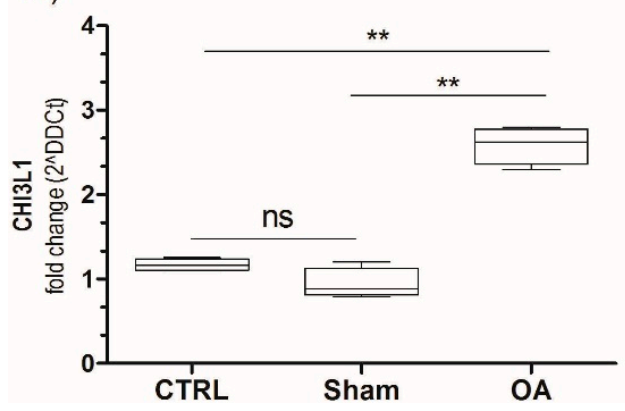

B)

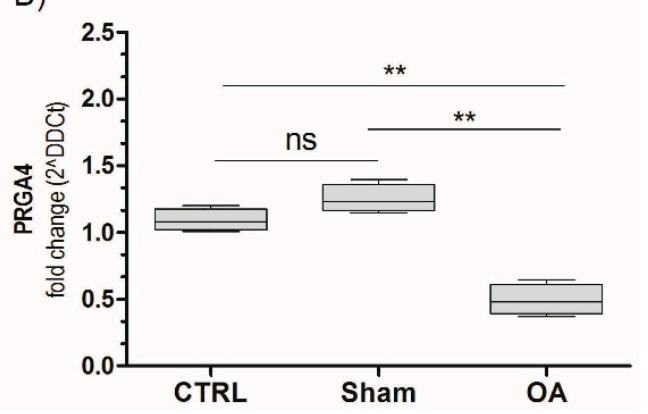

Figure 6. CHI3L1 (A) and PRG4 (B) mRNA expression in osteoarthritic rat cartilage model. Expression levels of CHI3L1 and PRG4 (lubricin) in cartilage of osteoarthritis rats. Total RNA was extracted as indicated in Materials and Method and CHI3L1/PRG4 expression was measured by real-time PCR. Data are expressed as mean $\pm \mathrm{SD}$ of at least three independent experiments. ${ }^{* *} p<0.001$, compared to sham and control. CTRL: control; ns: not significant.

\section{Discussion}

In this study the histomorphometric results in control and sham groups (without ACLT), showed an intact and normal cartilage structure without signs of cartilage degeneration (Kellgren-Lawrence OA severity scores, Grade 0; Kraus' modified Mankin score, Grade 0; Histopathology OARSI system, Grade 0), whilst in the OA group (with ACLT) cartilage showed more serious pathological changes, as horizontal cleavage tears or flaps and deep lesions, confirming moderate OA (Kellgren-Lawrence OA severity scores, Grade 2; Kraus' modified Mankin score, Grade 2; Histopathology OARSI system, Grades 2/3). The development of articular degenerative processes in OA group was clear and significantly different from the control groups, as confirmed by the Kellgren-Lawrence OA severity scores, Kraus' modified Mankin score and histopathology OARSI system. These results were supported by immunohistochemistry, double-staining and mRNA examination. In this study the immunoexpression and the mRNA levels of lubricin increased in normal cartilage and decreased in OA cartilage, while the immunoexpression and the mRNA levels of CHI3L1 increased in OA cartilage and decreased in normal cartilage (Figure 7). We found a negative correlation between the expression of the chondroprotective lubricin and the pro-inflammatory CHI3L1 in normal cartilage and in an experimental model of OA cartilage.

$\mathrm{OA}$ is a disease with a high incidence and prevalence, with an expected increase in the number of affected individuals, particularly due to the aging of the population, but also due to the increasing prevalence of obesity and a sedentary lifestyle [16,17]. It is becoming clear that articular tissues other than cartilage play an important role in the process of OA; it is regarded as a whole joint disease. In the last decades the interest of biochemists and biologists focused on glycoproteins and it is well known that there are many biomarkers and glycoproteins involved in the OA pathophysiological process [18-23]. This improved attention is partly due to the fact that glycoproteins were revealed to be abundant in living organisms [24]. Many glycoproteins have structural functions, form connective tissues such as collagen, and are used as protective agents and lubricants [24-26]. These molecules are composed of a peptide chain with one or more carbohydrate moieties linked $N$-glycosidically or $O$-glycosidically to their constituent protein. According to these structures, glycoproteins are divided in two categories. Fine structural differences within these broader categories, account for the variety of functions among glycoproteins. Specific enzymes regulate glycoprotein synthesis and degradation [24-26]. 


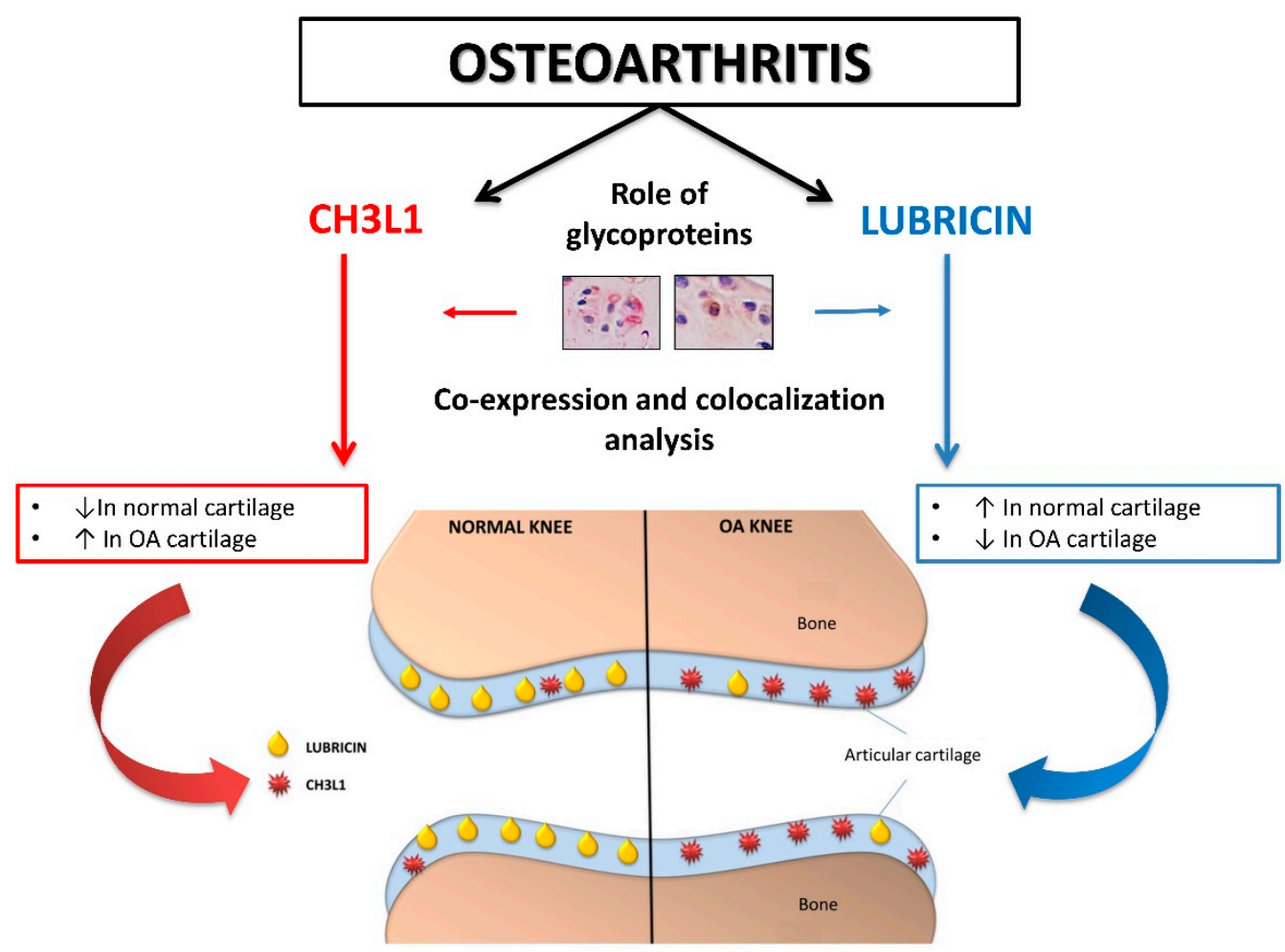

Figure 7. Graphical representation of the involvement of two glycoproteins (CHI3L1 and lubricin) in normal and osteoarthritic articular cartilage. Red arrows (CHI3L1 pathway); Blue arrows (lubricin pathway).

In this study we investigated the co-expression and the co-localization of two fundamental glycoproteins $[27,28]$ involved in cartilage, CHI3L1 and lubricin. CHI3L1, or YKL-40, is a glycoprotein produced by articular chondrocytes, synoviocytes and macrophages. Serum and SF (Synovial fluid) levels of CHI3L1 are increased in inflammatory diseases and correlate with the grade of cartilage degeneration in rheumatoid arthritis. CHI3L1 is a candidate auto antigen in rheumatoid arthritis related to the ability of cells to respond to and cope with variations in their environment [29]. Recently, authors stated that chondrocytes of human osteoarthritic cartilage secrete the inflammation associated chitolectin CHI3L1 [30]. CHI3L1 is a major secretory protein of human chondrocytes in cell culture. CHI3L1 mRNA is not detectable in normal human cartilage, though it is expressed in cartilage from patients with rheumatoid arthritis [31]. Moreover, CHI3L1 levels in SF but not serum were independently and positively related to clinical findings, such as pain and physical disability, in knee OA patients; thus it could represent a potential biomarker of severity of OA [32].

Another important glycoprotein is represented by lubricin. Lubricin is a chondroprotective, mucinous glycoprotein, the product of the proteoglycan 4 (PRG4) gene [33]. It has been found in several tissues including the synovial membranes and SF [34], the superficial zone of articular cartilage [35], tendon and ligament [36], disc and meniscus $[37,38]$. The lubricin role is maintaining joint integrity. Lubricin is a lubricating glycoprotein present in SF, specifically produced and expressed by articular chondrocytes of the superficial zone. It is recognized as playing a major protective role, preventing cartilage wear and synovial cell adhesion and proliferation and reducing the amount of friction of the articular cartilage surface [39]. It has been also demonstrated that physical activity promotes the expression of lubricin and attenuates the cartilage degeneration process, suggesting again its important role in chondroprotection [40-42]. Indeed, the lack of lubricin secretion may be involved in the pathology of OA. Authors demonstrated that considerable negative regulation of lubricin, as well as of other proteoglycans and SF biomarkers, develops in the human knee meniscus and anterior cruciate ligament (ACL) in the acute phase of joint injury, highlighting its involvement in articular 
injury $[38,43]$. When recombinant lubricin was injected in injured joints, in a study on animal model of OA, its improved chondroprotection, suggesting its potential use in new approaches for the treatment of OA and other cartilage disease [44-48].

The results of the present research are in accordance with our previous studies $[9,35,39]$. Here, we demonstrated for the first time the co-expression and the co-localization of these two glycoproteins in in vivo normal cartilage, and in an experimental model of OA cartilage. CHI3L1 overexpression was found in chondrocytes from the OA group mainly in the middle and deep zone of the cartilage rather than the superficial zone, while it was weakly expressed in cartilage from the superficial, middle and deep zones of control and sham group cartilage. In contrast, in the control and in the sham groups, lubricin overexpression was found mainly in chondrocytes from the superficial and middle zone of the cartilage rather than the deep zone, while it was weakly expressed in cartilage from superficial, middle and deep zone of osteoarthritic cartilage.

Our results indicate that $\mathrm{CHI} 3 \mathrm{~L} 1$ and lubricin might be considered as potential natural agents for providing therapeutic protective effects in joint inflammation, and/or may promote cartilage preservation in degenerative disorders of articular cartilage.

The findings of our study suggest that the two glycoproteins, CHI3L1 and lubricin, could be functionally associated with the development of OA, in particular with grade $2 / 3$ of OA evidenced in histomorphometric analysis of our samples, and could be used as biomarker matches, suggesting that in the future they could be helpful to stage the severity and progression of the disease.

\section{Materials and Methods}

\subsection{Breeding and Housing of Animals}

Thirty 3-month-old healthy male Wistar Outbred Rats (Charles River Laboratories, Milan, Italy), with an average body weight of $160 \pm 80 \mathrm{~g}$, were used for this study. Rats were housed in polycarbonate cages (cage dimensions: $\left.10.25^{\prime \prime} \mathrm{W} \times 18.75^{\prime \prime} \mathrm{D} \times 8^{\prime \prime} \mathrm{H}\right)$ at controlled temperature $\left(20-23^{\circ} \mathrm{C}\right)$ and humidity during the whole period of the research, with free access to water and food and photoperiod of $12 \mathrm{~h}$ light/dark. Surgical procedures for ACLT were performed in accordance with the method previously described $[1,9,49]$. The ACLT surgery procedure was made under total anesthesia, $30 \mathrm{mg} / \mathrm{kg}$ Zoletil $100+$ altadol $5 \mathrm{mg} / \mathrm{kg}+$ maintenance mixture of $\mathrm{O}_{2}$ and isoflurane $2 \%-2.5 \%$, (Vibrac, Milan, Italy. The anterior portion of the left hind limb was shaved with an electric clipper, and cleaned with povidone iodine (Sceptre Medical, New Delhi, India). The skin around the knee cap was vertically incised along the medial border of the knee cap. The patella was displaced laterally to expose the anterior cruciate ligament. Then, the anterior cruciate ligament was cut with surgical scissors without injury to the cartilage of the tibia. The patella was then replaced back, and the fascia and skin were closed with a 3-0 polydioxanone suture. A single dose of antibiotic Convenia ${ }^{\circledR} 0.1 \mathrm{~mL} / \mathrm{kg}$, (Vibrac, Milan, Italy) cream was applied to avoid postoperative infection. After surgery, free cage movement without joint immobilization was permitted to all animals. The 30 animals were divided in two groups: the control group without ACLT (10 rats) and the OA group with ACLT (20 rats). The control group was composed of two subgroups: control normal group (5 rats) without surgical treatment and sham-operated control group (5 rats), receiving the same surgical procedure as the experimental group, without ACLT. The OA group instead consisted of 20 rats submitted to ACLT surgical treatment inducing the OA model. During the experiment the possible suffering of the animals was monitored through the clinical conditions of the animal (fur appearance, weight, consumption of food and water, lameness) evaluated once a day. The animals from all groups at 2 months after the surgical procedures were sacrificed by intracardial Pentothal ${ }^{\circledR}$ injection $30-40 \mathrm{mg} / \mathrm{kg}$ (Biochemie, Kundl, Austria); under Furane $2 \%{ }^{\circledR}$-narcosis (Abbott Laboratories, Maidenhead, Berks, UK). The pre-operative examinations included physical examination, photographical examination and X-ray microtomography imaging (Figure 8C). The radiographic analyses demonstrating the joint pathology were preliminary to explantation of both femurs that were cleaned from soft tissues and used to perform histomorphometric evaluations 
(Figure 8A,B,D). Each sample of articular cartilage, from the three groups, was divided in fragments in order to perform histological, immunohistochemical and gene expression analyses. All procedures conformed to the guidelines of the Institutional Animal Care and Use Committee (I.A.C.U.C.) of the University of Catania (Protocol n. 125 of the 1 July 2011, Italian Ministry of Health). The experiments were conducted in accordance with the European Community Council Directive (86/609/EEC) and the Italian Animal Protection Law (116/1992).
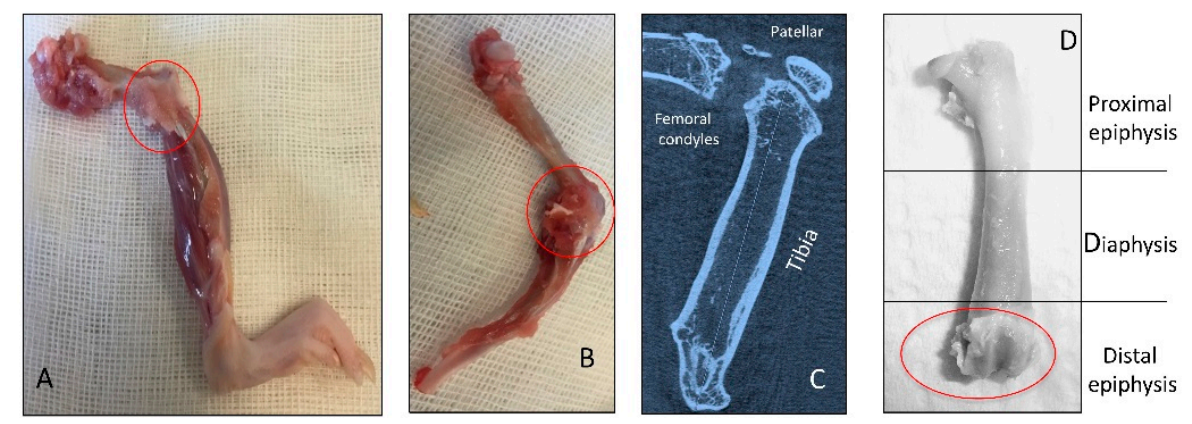

Figure 8. Representation of in vivo explanted knee join bones (red circles). (A,B) Knee OA bones joint after explantation, not cleaned of soft tissues; (C) X-ray microtomography imaging of the OA knee bones joint; (D) OA femur cleaned of soft tissues.

\subsection{Radiographic Analysis}

The pre-operative examinations included physical examination, photographical examination and X-ray microtomography imaging (Bruker, Milan, Italy). We chose our samples in accordance with the radiographic OA severity evaluated by the Kellgren and Lawrence score [50] to classify the severity of knee OA that showed radiographic worsening over time. Two blinded investigators (two anatomical morphologists) made the analyses, and the evaluations were assumed correct if no statistically significant difference was showed between the investigators. The Kellgren and Lawrence system provides a score of severity from 0 to 4 : Grade 0 , no radiographic features of OA are present; Grade 1, doubtful joint space narrowing (JSN) and possible osteophytic lipping; Grade 2, definite osteophytes and possible JSN on anteroposterior weight-bearing radiograph; Grade 3, multiple osteophytes, definite JSN, sclerosis, possible bony deformity; Grade 4, large osteophytes, marked JSN, severe sclerosis and definite bony deformity. The inter-observer variability between two observers for the Kellgren-Lawrence score showed a similar good intra-class correlation coefficient (ICC > 0.84). Repeat scoring by investigators showed very good agreement (ICC > 0.90).

\subsection{Histomorphometric Analysis}

The femurs explantation procedure and the subsequent cleaning of soft tissues was performed as previously described [51]. Samples from all rats (both medial and lateral femoral condyles of untreated and surgically treated animals) were used for the histomorphometric analysis. Histomorphometry was performed with image analysis, Kontron KS 300 software (Kontron Electronics, Eching bei Munchen, Germany) by three blinded investigators (two anatomical morphologists and one histologist). Evaluations were assumed correct if there were no statistically significant differences between the investigators. Fifteen fields randomly selected from each section were analyzed. The semi-quantitative grading criteria of macroscopic Kraus' modified Mankin score [52,53] and microscopic histopathology OARSI system [54,55] were used. The inter-observer variability among 3 observers for the Mankin system showed a good intra-class correlation coefficient (ICC > 0.92) as for the OARSI system (ICC > 0.89). Repeat scoring by investigators showed very good agreement (ICC >0.94).

The Kraus' modified Mankin score provides grades from 0 to 4: Grade 0, normal cartilage; Grade 1, minimal articular damage; Grade 2, articular cartilage damage affecting up to $30 \%$ of the articular 
surface; Grade 3, loss of up to $50 \%$ of the articular cartilage; Grade 4, severe loss of cartilage affecting more than $50 \%$ of the articular surface.

The Histopathology OARSI system provides grades from 0 to 6: Grade 0, normal articular cartilage; Grade 1, intact surface; Grade 2, surface discontinuity; Grade 3, vertical fissures extending into mid zone; Grade 4, erosion; Grade 5, denudation; Grade 6, deformation.

\subsection{Histology and Histochemistry Analysis}

Some fragments of articular cartilage samples were fixed in 10\% neutral buffered-formalin (Bio-Optica, Milan, Italy), following overnight washing and routinely embedded in paraffin as previously described [1]. Samples were positioned in the cassettes in the same direction after wax infiltration. A rotary manual microtome (Leica RM2235, Milan, Italy) was used to cut 4-5 $\mu \mathrm{m}$ thick sections from paraffin blocks that were mounted on silane-coated slides (Menzel-Gläser, Braunschweig, Germany) and stored at room temperature. After dewaxing in xylene, the slides were hydrated using graded ethanol, and stained for histological evaluation by Hematoxylin and Eosin (Figure 9C,F-H) and Masson's Trichrome (Figure 9B,D) (Bio-Optica) staining for cell identification and the detection of structural alterations. Then the slides were analysed through toluidine blue staining (Figure 9A,E) (Fluka, St. Louis, MO, USA) to assess synthesis of sulfated glycosaminoglycan (GAG) containing proteoglycans (basing on the intensity of staining), in order to evaluate the experimental induction of OA according to the histopathology OARSI system. The samples were examined with a Zeiss Axioplan light microscope (Carl Zeiss, Oberkochen, Germany) and a digital camera (AxioCam MRc5, Carl Zeiss) was used to take the pictures.
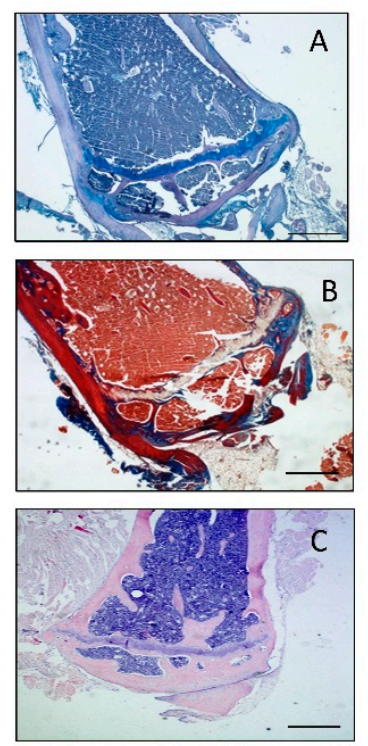
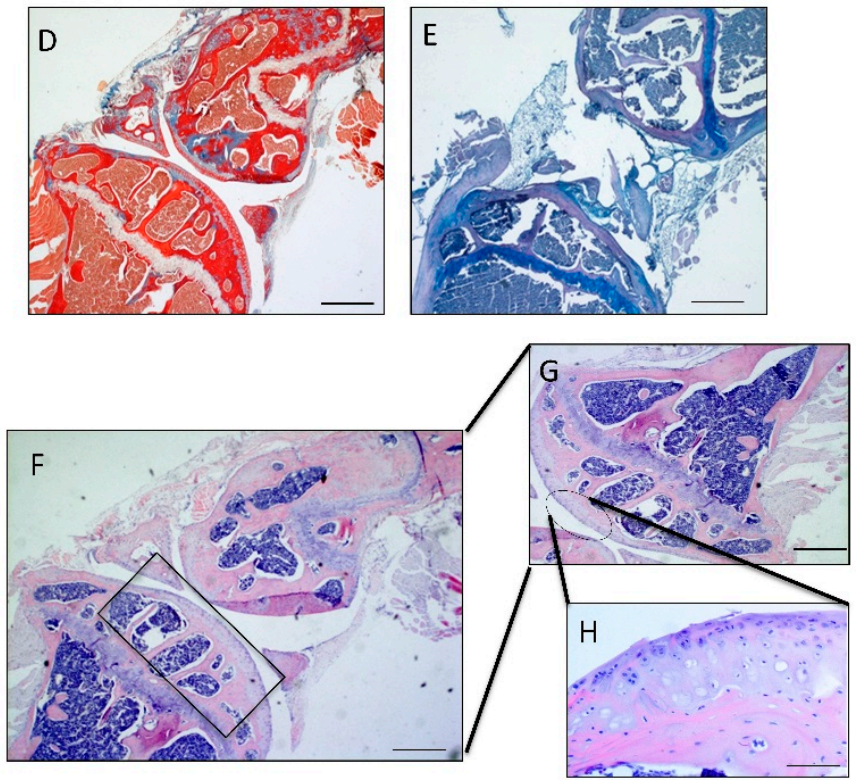

Figure 9. (A) Micrograph of the OA distal epiphysis of the femur stained with Toluidine Blue, Magnification $\times 10$; (B) Micrograph of the OA distal epiphysis of the femur stained with Masson's Trichrome, Magnification $\times 10$; (C) Micrograph of the OA distal epiphysis of the femur stained with Hematoxylin and Eosin, Magnification $\times 10$; (D) Micrograph of the OA knee joint stained with Masson's Trichrome, Magnification $\times 5$; (E) Micrograph of the OA knee joint stained with Toluidine Blue, Magnification $\times 5$; (F) Micrograph of the OA knee joint stained with Hematoxylin and Eosin, Magnification $\times 5$; (G) Magnification of $\mathrm{J} \times 10$; $(\mathbf{H})$ Magnification $\times 20$, Scale bars: $100 \mu \mathrm{m}$.

\subsection{Immunohistochemistry (IHC) Analysis}

Some fragments of articular cartilage were processed for immunohistochemical analysis was previously described [56]. Briefly, after dewaxing in xylene, the slides were hydrated through graded 
ethanol and incubated for $30 \mathrm{~min}$ in $0.3 \% \mathrm{H}_{2} \mathrm{O}_{2} /$ methanol to quench endogenous peroxidase activity and then rinsed for 20 min with phosphate-buffered saline (PBS; Bio-Optica). The sections were then heated $(5 \mathrm{~min} \times 3)$ in capped polypropylene slide-holders with citrate buffer $(10 \mathrm{mM}$ citric acid, 0.05\% Tween 20, pH 6.0; Bio-Optica), using a microwave oven (750 W) to unmask antigenic sites. The blocking step to prevent non-specific binding of the antibody was performed before application of the primary antibody with 5\% bovine serum albumin (BSA, Sigma, Milan, Italy) in PBS for $1 \mathrm{~h}$ in a moist chamber. After blocking, the sections were incubated overnight at $4{ }^{\circ} \mathrm{C}$ with goat polyclonal GP-39 antibody (CHI3L1), work dilution in PBS 1:100 (sc-30465, Santa Cruz Biotechnology, Inc., Dallas, TX, USA) and with rabbit polyclonal anti-lubricin antibody (ab28484; Abcam, Cambridge, UK), diluted 1:100 in PBS (Bio-Optica) for $10 \mathrm{~min}$. Immune complexes were then treated with a biotinylated link antibody (HRP-conjugated anti-goat and anti-rabbit were used as secondary antibodies) and then detected with peroxidase labeled streptavin, both incubated for $10 \mathrm{~min}$ at room temperature (LSAB+ System-HRP, K0690, Dako, Denmark). The immunoreaction was visualized by incubating the sections for $2 \mathrm{~min}$ in a $0.1 \%$ 3,3'-diaminobenzidine and $0.02 \%$ hydrogen peroxide solution (DAB substrate Chromogen System; Dako, Denmark). The samples were lightly counterstained with Mayer's Hematoxylin (Histolab Products AB, Goteborg, Sweden) mounted in GVA mount (Zymed, Laboratories Inc., San Francisco, CA, USA) and observed with an Axioplan Zeiss light microscope (Carl Zeiss) and photographed with a digital camera (AxioCam MRc5, Carl Zeiss).

\subsection{Double Immuno-Staining Analysis}

Double staining was performed with the specific antibodies against lubricin (red) and CHI3L1 (brown) to investigate their expression and to assess their distribution in normal and osteoarthritic articular cartilage tissue. The procedure was performed according to the manufacturer's instructions using kit EnVision ${ }^{\mathrm{TM}}$ G/2 Doublestain System (Dako, Glostrup, Denmark), Rabbit/Mouse DAB+/Permanent Red (K5261; Dako, Glostrup, Denmark). Briefly, the slides were incubated with Dual Endogenous Enzyme Block solution (kit EnVision ${ }^{\mathrm{TM}} \mathrm{G} / 2$ Doublestain System), containing 0.5\% hydrogen peroxide, detergents, enzyme inhibitors and preservative, $\mathrm{pH} 2$, for 5 min before being rinsed with PBS (Bio-Optica) for $5 \mathrm{~min}$. After, the sections were incubated with goat polyclonal GP-39 antibody (CHI3L1; sc-30465, Santa Cruz Biotechnology, Inc.) working dilution 1:100 in PBS. Immune complexes were then treated with dextran polymer conjugated with horseradish peroxidase and affinity-isolated immunoglobulins, Polymer/HRP (kit EnVision ${ }^{\mathrm{TM}}$ G/2 Doublestain System), for $10 \mathrm{~min}$. Immunoreactivity was visualized by incubating the sections for $2 \mathrm{~min}$ in a $0.1 \% 3,3^{\prime}$-diaminobenzidine and $0.02 \%$ hydrogen peroxide solution (DAB+ Working Solution, prepared by thoroughly mixing $1 \mathrm{~mL}$ of DAB+ Substrate Buffer with 1 drop $(25-30 \mu \mathrm{L})$ of DAB+ Chromogen; kit EnVision ${ }^{\mathrm{TM}} \mathrm{G} / 2$ Doublestain System). Then the slides were treated with blocking solution, Doublestain Block (kit EnVision $^{\mathrm{TM}}$ G/2 Doublestain System), for $3 \mathrm{~min}$, before incubating them with the second rabbit polyclonal anti-lubricin antibody (ab28484; Abcam), diluted 1:100 in PBS (Bio-Optica) for 10 min. The slides were then incubated with dextran polymer coupled with secondary antibodies against goat and rabbit immunoglobulins, Rabbit/Mouse link (kit EnVision ${ }^{\mathrm{TM}} \mathrm{G} / 2$ Doublestain System), for $10 \mathrm{~min}$ and after, with dextran polymer conjugated with alkaline phospatase and affinity-isolated immunoglobulins, Polymer/AP (kit EnVision ${ }^{\mathrm{TM}} \mathrm{G} / 2$ Doublestain System), for 10 min. At this point, the immunoreactivity for the second antibody was visualized by using the Permanent Red Working Solution, prepared by thoroughly mixing 100 parts of Permanent Red Substrate Buffer with 1 part of Permanent Red Chromogen (kit EnVision ${ }^{\mathrm{TM}}$ G/2 Doublestain System), for $10 \mathrm{~min}$. The sections were lightly counterstained with Mayer's hematoxylin (Histolab Prod-ucts AB, Göteborg, Sweden) and mounted in Dako Glycergel ${ }^{\mathrm{TM}}$ Mounting Medium (C0563; Dako, Glostrup, Denmark). The stained slides were observed with an Axioplan Zeiss light microscope (Carl Zeiss) and photographed with a digital camera (AxioCam MRc5, Carl Zeiss). 


\subsection{Evaluation of Immunohistochemistry}

The CHI3L1 and lubricin-staining status were identified as either negative or positive. As previously described, immunohistochemical staining was defined positive if brown or red chromogens were detected on the edge of the hematoxylin-stained cell nucleus, within the cytoplasm or in the membrane [56]. Light microscopy was used to evaluate stain intensity and the percentage of immunopositive cells. Intensity of staining (IS) was evaluated on a 4 grades scale (0-4), as following: no detectable staining $=0$, weak staining $=1$, moderate staining $=2$, strong staining $=3$, very strong staining $=4$. Three investigators ( 2 anatomical morphologists and one histologist) independently evaluated the percentage of antibodies immunopositive cells through the five categories of Extent Score (ES): $<5 \%(0) ; 5 \%-30 \%(+) ; 31 \%-50 \%(++) ; 51 \%-75 \%(+++)$, and $>75 \%(++++)$. Counting was performed under Zeiss Axioplan light microscope at $\times 200$ magnification. If disputes concerning the interpretation occurred, the case was revised to reach a unanimous agreement, as previously described [9]. A digital camera (Canon, Tokyo, Japan) at $\times 20, \times 40$ and $\times 60$ magnifications was used to take digital pictures. In this study positive controls, consisted of rat cartilage tissue, and negative control sections, treated with PBS without the primary antibodies, were performed to test the specific reaction of primary antibodies used at a protein level. Positive immunolabeling for antibodies were nuclear/cytoplasmic.

\subsection{Computerized Morphometric Measurements and Image Analysis}

Image analysis software (AxioVision Release 4.8.2-SP2 Software, Carl Zeiss Microscopy GmbH, Jena, Germany), which quantifies the level of staining intensity of positive immunolabelling, was used to calculate the percentage area stained with CHI3L1 and lubricin antibodies in 15 fields, randomly selected from each section. Digital micrographs were taken using the Zeiss Axioplan light microscope (Carl Zeiss, using objective lens of magnification $\times 20$ i.e., total magnification 400) fitted with a digital camera (AxioCam MRc5, Carl Zeiss). Three blinded investigators ( 2 anatomical morphologists and one histologist) made the evaluations that were assumed to be correct if values have not statistically significant difference [57]. If disputes concerning interpretation occurred, unanimous agreement was reached after sample re-evaluation.

\subsection{RNA Isolation and Preparation}

Some fragments of articular cartilage were immersed in QIAzol (Qiagen, Mississauga, ON, Canada). After homogenization, total RNA was isolated using the Lipid Tissues Mini Kit (Qiagen, Mississauga, ON, Canada), according to the instructions of the manufacturer (Qiagen). RNA quantity was evaluated using the RiboGreen Assay (Molecular Probes, Burlington, ON, Canada), and RNA quality was assessed using an Agilent 2100 Bioanalyzer (Agilent, Palo Alto, CA, USA).

\subsection{Gene Expression Analysis by Real-Time PCR ( $q R T-P C R)$}

Total RNA extracted (500-800 ng per sample) from articular cartilage was reverse-transcribed with RevertAid First Strand cDNA Synthesis kit (Thermo Scientific, Milan, Italy) in a $20 \mu \mathrm{L}$ reaction solution. Quantitative RT-PCR was performed using one-twentieth of the RT products and platinum SYBR Green qPCR SuperMix UDG with Rox (Invitrogen Life Technologies, Milan, Italy). The primers used are shown in Table 2. The reaction was followed by a melting curve protocol according to the specifications of the ABI 7900 instrument (Applied Biosystems, Foster City, CA, USA). Rat $\beta$-Actin (ACTB) was used as a housekeeping gene for normalization. Data are presented as mean \pm SD of at least three independent experiments. Differences were analyzed by Student's $t$ test, with $p<0.01$ being considered statistically significant. 
Table 2. Primers used in gene expression analysis by real-time PCR (qRT-PCR). PRG4: proteoglycan 4; ACTB: $\beta$-Actin; Ta: thymine and adenine.

\begin{tabular}{ccccc}
\hline Primers & Forward & Reverse & Ta & Size \\
\hline PRG4 & CTACAACAGCTTCTGCGAAGAA & GATTTGGGTGAACGTTTGGTGG & 60 & 117 \\
CHI3L1 & GAGCTGCTTCCCAGATGCCC & CATGCCATACAGGGTTACGTC & 60 & 121 \\
ACTB & CATGTACGTAGCCATCCAGG & CTCTCAGCTGTGGTGGTGAA & 57 & 225 \\
\hline
\end{tabular}

\subsection{Statistical Analysis}

Statistical analysis was performed using GraphPad Instat ${ }^{\circledR}$ Biostatistics version 3.0 software (GraphPad Software, Inc., La Jolla, CA, USA). Data were tested for normality with the Kolmogorov-Smirnov test. All variables were normally distributed. Student's $t$ test was used for comparisons between two means, while analysis of variance (ANOVA) and Bonferroni's test were used for comparison between more than two groups. $p$-values of less than $0.05(p<0.05)$ was considered statistically significant; $p$-values of less than $0.01(p<0.01)$ was considered highly statistically significant; $p$-values of less than $0.001(p<0.001)$ was considered extremely statistically significant. Data are presented as the mean \pm SEM. Cohen's $k$ was applied to measure the agreement between the two-blinded observers and averaged to evaluate overall agreement.

\section{Conclusions}

The therapeutic management of OA remains a challenge for physicians. In this setting the basic sciences are involved in finding information regarding the pathophysiological process of this common and severe disease. In this study we highlighted a possible cross-link between two pathological aspects of the inflammatory process and the altered lubricating ability occurring in the joint tissue following chronic disease, such as OA. Our findings might motivate further studies about the link between the two important aspects of OA, inflammatory process through the study of CHI3L1 and lubricating ability through the study of lubricin. However, further studies are needed to understand the exact mechanisms in which these molecules are involved in order to design therapies or treatments to prevent or attenuate the osteoarthritic process.

Acknowledgments: This study was supported by a Grant-in-Aid provided by University Research Project FIR 2014-2016, (cod. 314509), University of Catania, Italy. The authors would like to thank Iain Halliday for commenting and making corrections to the paper and Ana Valdes from Nottingham University for her important suggestions.

Author Contributions: All authors have made substantial intellectual contributions to the conception and design of the study as well as data acquisition, analysis and interpretation. Marta Anna Szychlinska, Francesca Maria Trovato, Michelino Di Rosa and Lidia Puzzo carried out the experimental in vivo and in vitro work, study execution and literature research. Lucia Malaguarnera, Rosy Leonardi and Paola Castrogiovanni contributed to data collection, study execution and provided technical assistance. Giuseppe Musumeci conceived the study design, supervised the manuscript writing, planning and editing, coordinated the execution of experimental procedures and the analysis and discussion of results. All authors contributed to data interpretation and manuscript preparation. All authors approved the final submitted version.

Conflicts of Interest: The authors declare no conflict of interest.

\section{Abbreviations}

\begin{tabular}{ll}
\hline OA & Osteoarthritis \\
GP-39 & Glycoprotein 39 \\
CHI3L1 YKL-40 & Chitinase 3-like-1 \\
SF & Synovial fluid \\
SZP & Superficial zone protein \\
PRG4 & Proteoglycan 4 \\
ACLT & Anterior cruciate ligament transection \\
\hline
\end{tabular}




\section{References}

1. Giunta, S.; Castorina, A.; Marzagalli, R.; Szychlinska, M.A.; Pichler, K.; Mobasheri, A.; Musumeci, G. Ameliorative effects of PACAP against cartilage degeneration. Morphological, immunohistochemical and biochemical evidence from in vivo and in vitro models of rat osteoarthritis. Int. J. Mol. Sci. 2015, 16, 5922-5944. [CrossRef] [PubMed]

2. Mobasheri, A.; Matta, C.; Zákány, R.; Musumeci, G. Chondrosenescence: Definition, hallmarks and potential role in the pathogenesis of osteoarthritis. Maturitas 2015, 80, 237-244. [CrossRef] [PubMed]

3. Musumeci, G.; Aiello, F.C.; Szychlinska, M.A.; di Rosa, M.; Castrogiovanni, P.; Mobasheri, A. Osteoarthritis in the XXIst century: Risk factors and behaviours that influence disease onset and progression. Int. J. Mol. Sci. 2015, 16, 6093-6112. [CrossRef] [PubMed]

4. Musumeci, G.; Szychlinska, M.A.; Mobasheri, A. Age-related degeneration of articular cartilage in the pathogenesis of osteoarthritis: Molecular markers of senescent chondrocytes. Histol. Histopathol. 2015, 30, 1-12. [CrossRef] [PubMed]

5. Di Rosa, M.; Malaguarnera, G.; de Gregorio, C.; D’Amico, F.; Mazzarino, M.C.; Malaguarnera, L. Modulation of chitotriosidase during macrophage differentiation. Cell. Biochem. Biophys. 2013, 66, 239-247. [CrossRef] [PubMed]

6. Di Rosa, M.; de Gregorio, C.; Malaguarnera, G.; Tuttobene, M.; Biazzo, F.; Malaguarnera, L. Evaluation of AMCase and CHIT-1 expression in monocyte macrophages lineage. Mol. Cell. Biochem. 2013, 374, 73-80. [CrossRef] [PubMed]

7. Di Rosa, M.; Malaguarnera, G.; de Gregorio, C.; Drago, F.; Malaguarnera, L. Evaluation of CHI3L-1 and CHIT-1 expression in differentiated and polarized macrophages. Inflammation 2013, 36, 482-492. [CrossRef] [PubMed]

8. Väänänen, T.; Koskinen, A.; Paukkeri, E.L.; Hämäläinen, M.; Moilanen, T.; Moilanen, E.; Vuolteenaho, K. YKL-40 as a novel factor associated with inflammation and catabolic mechanisms in osteoarthritic joints. Mediat. Inflamm. 2014, 2014. [CrossRef] [PubMed]

9. Di Rosa, M.; Szychlinska, M.A.; Tibullo, D.; Malaguarnera, L.; Musumeci, G. Expression of CHI3L1 and CHIT1 in osteoarthritic rat cartilage model. A morphological study. Eur. J. Histochem. 2014, 58. [CrossRef] [PubMed]

10. Jay, G.D.; Fleming, B.C.; Watkins, B.A.; McHugh, K.A.; Anderson, S.C.; Zhang, L.X.; Teeple, E.; Waller, K.A.; Elsaid, K.A. Prevention of cartilage degeneration and restoration of chondroprotection by lubricin tribosupplementation in the rat following anterior cruciate ligament transection. Arthritis Rheum. 2010, 62, 2382-2391. [CrossRef] [PubMed]

11. Elsaid, K.A.; Zhang, L.; Waller, K.; Tofte, J.; Teeple, E.; Fleming, B.C.; Jay, G.D. The impact of forced joint exercise on lubricin biosynthesis from articular cartilage following ACL transection and intra-articular lubricin's effect in exercised joints following ACL transection. Osteoarthr. Cartil. 2012, 20, 940-948. [CrossRef] [PubMed]

12. Leonardi, R.; Musumeci, G.; Sicurezza, E.; Loreto, C. Lubricin in human temporomandibular joint disc: An immunohistochemical study. Arch. Oral Biol. 2012, 57, 614-619. [CrossRef] [PubMed]

13. Musumeci, G.; Loreto, C.; Carnazza, M.L.; Strehin, I.; Elisseeff, J. OA cartilage derived chondrocytes encapsulated in poly(ethylene glycol) diacrylate (PEGDA) for the evaluation of cartilage restoration and apoptosis in an in vitro model. Histol. Histopathol. 2011, 26, 1265-1278. [PubMed]

14. Loeser, R.F. Osteoarthritis year in review 2013: Biology. Osteoarthr. Cartil. 2013, 21, 1436-1442. [CrossRef] [PubMed]

15. Ruan, M.Z.; Erez, A.; Guse, K.; Dawson, B.; Bertin, T.; Chen, Y.; Jiang, M.M.; Yustein, J.; Gannon, F.; Lee, B.H. Proteoglycan 4 expression protects against the development of osteoarthritis. Sci. Transl. Med. 2013, 5. [CrossRef] [PubMed]

16. Johnstone, B.; Alini, M.; Cucchiarini, M.; Dodge, G.R.; Eglin, D.; Guilak, F.; Madry, H.; Mata, A.; Mauck, R.L.; Semino, C.E.; et al. Tissue engineering for articular cartilage repair-the state of the art. Eur. Cell Mater. 2013, 2, 248-267.

17. Musumeci, G.; Loreto, C.; Carnazza, M.L.; Coppolino, F.; Cardile, V.; Leonardi, R. Lubricin is expressed in chondrocytes derived from osteoarthritic cartilage encapsulated in poly(ethylene glycol) diacrylate scaffold. Eur. J. Histochem. 2011, 55, 162-168. [CrossRef] [PubMed] 
18. Musumeci, G.; Castrogiovanni, P.; Mazzone, V.; Szychlinska, M.A.; Castorina, S.; Loreto, C. Histochemistry as a unique approach for investigating normal and osteoarthritic cartilage. Eur. J. Histochem. 2014, 58, 107-111. [CrossRef] [PubMed]

19. Tsukamoto, I.; Akagi, M.; Inoue, S.; Yamagishi, K.; Mori, S.; Asada, S. Expressions of local renin-angiotensin system components in chondrocytes. Eur. J. Histochem. 2014, 58. [CrossRef] [PubMed]

20. Shibata, S.; Sakamoto, Y.; Baba, O.; Qin, C.; Murakami, G.; Cho, B.H. An immunohistochemical study of matrix proteins in the craniofacial cartilage in midterm human fetuses. Eur. J. Histochem. 2013, 57. [CrossRef] [PubMed]

21. Loreto, C.; Leonardi, R.; Musumeci, G.; Pannone, G.; Castorina, S. An ex vivo study on immunohistochemical localization of MMP-7 and MMP-9 in temporomandibular joint discs with internal derangement. Eur. J. Histochem. 2013, 57. [CrossRef] [PubMed]

22. Lehmann, M.; Martin, F.; Mannigel, K.; Kaltschmidt, K.; Sack, U.; Anderer, U. Three dimensional scaffold-free fusion culture: The way to enhance chondrogenesis of in vitro propagated human articular chondrocytes. Eur. J. Histochem. 2013, 57. [CrossRef] [PubMed]

23. Musumeci, G.; Castrogiovanni, P.; Trovato, F.M.; Weinberg, A.M.; Al-Wasiyah, M.K.; Alqahtani, M.H.; Mobasheri, A. Biomarkers of chondrocyte apoptosis and autophagy in osteoarthritis. Int. J. Mol. Sci. 2015, 16, 20560-20575. [CrossRef] [PubMed]

24. Halper, J.; Kjaer, M. Basic components of connective tissues and extracellular matrix: Elastin, fibrillin, fibulins, fibrinogen, fibronectin, laminin, tenascins and thrombospondins. Adv. Exp. Med. Biol. 2014, 802, 31-47. [PubMed]

25. Olson, S.A.; Horne, P.; Furman, B.; Huebner, J.; Al-Rashid, M.; Kraus, V.B.; Guilak, F. The role of cytokines in posttraumatic arthritis. J. Am. Acad. Orthop. Surg. 2014, 22, 29-37. [CrossRef] [PubMed]

26. Halper, J. Proteoglycans and diseases of soft tissues. Adv. Exp. Med. Biol. 2014, 802, 49-58. [PubMed]

27. Daniel, M. Boundary cartilage lubrication: Review of current concepts. Wien. Med. Wochenschr. 2014, 164, 88-94. [CrossRef] [PubMed]

28. Zivanović, S.; Rackov, L.P.; Vojvodić, D.; Vucetić, D. Human cartilage glycoprotein 39—Biomarker of joint damage in knee osteoarthritis. Int. Orthop. 2009, 33, 1165-1170. [CrossRef] [PubMed]

29. Baeten, D.; Steenbakkers, P.G.; Rijnders, A.M.; Boots, A.M.; Veys, E.M.; de Keyser, F. Detection of major histocompatibility complex/human cartilage GP-39 complexes in rheumatoid arthritis synovitis as a specific and independent histologic marker. Arthritis Rheum. 2004, 50, 444-451. [CrossRef] [PubMed]

30. Einarsson, J.M.; Bahrke, S.; Sigurdsson, B.T.; Ng, C.H.; Petersen, P.H.; Sigurjonsson, O.E.; Jonsson, H., Jr.; Gislason, J.; Thormodsson, F.R.; Peter, M.G. Partially acetylated chitooligosaccharides bind to YKL-40 and stimulate growth of human osteoarthritic chondrocytes. Biochem. Biophys. Res. Commun. 2013, 434, 298-304. [CrossRef] [PubMed]

31. Volck, B.; Ostergaard, K.; Johansen, J.S.; Garbarsch, C.; Price, P.A. The distribution of YKL-40 in osteoarthritic and normal human articular cartilage. Scand. J. Rheumatol. 1999, 28, 171-179. [PubMed]

32. Guan, J.; Liu, Z.; Li, F.; Feng, J.S.; Wang, H.J.; Chu, J.G.; Song, Y.Z.; Xie, L.; Ding, L.B. Increased synovial fluid YKL-40 levels are linked with symptomatic severity in knee osteoarthritis patients. Clin. Lab. 2015, 61, 991-997. [PubMed]

33. Musumeci, G.; Lo Furno, D.; Loreto, C.; Giuffrida, R.; Caggia, S.; Leonardi, R.; Cardile, V. Mesenchymal stem cells from adipose tissue which have been differentiated into chondrocytes in three-dimensional culture express lubricin. Exp. Biol. Med. 2011, 236, 1333-1341. [CrossRef] [PubMed]

34. Ludwig, T.E.; McAllister, J.R.; Lun, V.; Wiley, J.P.; Schmidt, T.A. Diminished cartilage-lubricating ability of human osteoarthritic synovial fluid deficient in proteoglycan 4: Restoration through proteoglycan 4 supplementation. Arthritis Rheum. 2012, 64, 3963-3971. [CrossRef] [PubMed]

35. Musumeci, G.; Loreto, C.; Leonardi, R.; Castorina, S.; Giunta, S.; Carnazza, M.L.; Trovato, F.M.; Pichler, K.; Weinberg, A.M. The effects of physical activity on apoptosis and lubricin expression in articular cartilage in rats with glucocorticoid-induced osteoporosis. J. Bone Miner. Metab. 2013, 31, 274-284. [CrossRef] [PubMed]

36. Leonardi, R.; Loreto, C.; Talic, N.; Caltabiano, R.; Musumeci, G. Immunolocalization of lubricin in the rat periodontal ligament during experimental tooth movement. Acta Histochem. 2012, 114, 700-704. [CrossRef] [PubMed] 
37. Leonardi, R.; Rusu, M.C.; Loreto, F.; Loreto, C.; Musumeci, G. Immunolocalization and expression of lubricin in the bilaminar zone of the human temporomandibular joint disc. Acta Histochem. 2012, 114, 1-5. [CrossRef] [PubMed]

38. Musumeci, G.; Loreto, C.; Carnazza, M.L.; Cardile, V.; Leonardi, R. Acute injury affects lubricin expression in knee menisci. An immunohistochemical study. Ann. Anat. 2013, 195, 151-158. [CrossRef] [PubMed]

39. Musumeci, G.; Trovato, F.M.; Pichler, K.; Weinberg, A.M.; Loreto, C.; Castrogiovanni, P. Extra-virgin olive oil diet and mild physical activity prevent cartilage degeneration in an osteoarthritis model. An "in vivo" and "in vitro" study on lubricin expression. J. Nutr. Biochem. 2013, 24, 2064-2075. [CrossRef] [PubMed]

40. Ogawa, H.; Kozhemyakina, E.; Hung, H.H.; Grodzinsky, A.J.; Lassar, A.B. Mechanical motion promotes expression of Prg4 in articular cartilage via multiple CREB-dependent, fluid flow shear stress-induced signaling pathways. Genes Dev. 2014, 28, 127-139. [CrossRef] [PubMed]

41. Musumeci, G.; Castrogiovanni, P.; Trovato, F.M.; Imbesi, R.; Giunta, S.; Szychlinska, M.A.; Loreto, C.; Castorina, S.; Mobasheri, A. Physical activity ameliorates cartilage degeneration in a rat model of aging: A study on lubricin expression. Scand. J. Med. Sci. Sports 2015, 25, e222-e230. [CrossRef] [PubMed]

42. Castrogiovanni, P.; Musumeci, G. Which is the Best Physical Treatment for Osteoarthritis? J. Funct. Morphol. Kinesiol. 2016, 1, 54-68. [CrossRef]

43. Catterall, J.B.; Stabler, T.V.; Flannery, C.R.; Kraus, V.B. Changes in serum and synovial fluid biomarkers after acute injury (NCT00332254). Arthritis Res. Ther. 2010, 12. [CrossRef] [PubMed]

44. Flannery, C.R. Novel therapies in OA. Curr. Drug Targets 2010, 11, 614-619. [CrossRef] [PubMed]

45. Flannery, C.R.; Zollner, R.; Corcoran, C.; Jones, A.R.; Root, A.; Rivera-Bermúdez, M.A.; Blanchet, T.; Gleghorn, J.P.; Bonassar, L.J.; Bendele, A.M.; et al. Prevention of cartilage degeneration in a rat model of osteoarthritis by intraarticular treatment with recombinant lubricin. Arthritis Rheum. 2009, 60, 840-847. [CrossRef] [PubMed]

46. Chevalier, X. Intraarticular treatments for osteoarthritis: New perspectives. Curr. Drug Targets 2010, 11, 546-560. [CrossRef] [PubMed]

47. Cui, Z.; Xu, C.; Li, X.; Song, J.; Yu, B. Treatment with recombinant lubricin attenuates osteoarthritis by positive feedback loop between articular cartilage and subchondral bone in ovariectomized rats. Bone 2015, 74, 37-47. [CrossRef] [PubMed]

48. Bao, J.P.; Chen, W.P.; Wu, L.D. Lubricin: A novel potential biotherapeutic approaches for the treatment of osteoarthritis. Mol. Biol. Rep. 2011, 38, 2879-2885. [CrossRef] [PubMed]

49. Musumeci, G.; Leonardi, R.; Carnazza, M.L.; Cardile, V.; Pichler, K.; Weinberg, A.M.; Loreto, C. Aquaporin 1 (AQP1) expression in experimentally induced osteoarthritic knee menisci: An in vivo and in vitro study. Tissue Cell 2013, 45, 145-152. [CrossRef] [PubMed]

50. Buck, R.J.; Wirth, W.; Dreher, D.; Nevitt, M.; Eckstein, F. Frequency and spatial distribution of cartilage thickness change in knee osteoarthritis and its relation to clinical and radiographic covariates-Data from the osteoarthritis initiative. Osteoarthr. Cartil. 2013, 21, 102-109. [CrossRef] [PubMed]

51. Pichler, K.; Loreto, C.; Leonardi, R.; Reuber, T.; Weinberg, A.M.; Musumeci, G. In rat with glucocorticoid-induced osteoporosis, RANKL is downregulated in bone cells by physical activity (treadmill and vibration stimulation training). Histol. Histopathol. 2013, 28, 1185-1196. [PubMed]

52. Mankin, H.J.; Dorfman, H.; Lippiello, L.; Zarins, A. Biochemical and metabolic abnormalities in articular cartilage from osteo-arthritic human hips. J. Bone Jt. Surg. 1971, 53, 523-537.

53. Kraus, V.B.; Huebner, J.L.; Stabler, T.; Flahiff, C.M.; Setton, L.A.; Fink, C.; Vilim, V.; Clark, A.G. Ascorbic acid increase the severity of spontaneous knee osteoarthritis in a guinea pig model. Arthritis Rheum. 2004, 50, 1822-1831. [CrossRef] [PubMed]

54. Pauli, C.; Grogan, S.P.; Patil, S.; Otsuki, S.; Hasegawa, A.; Koziol, J.; Lotz, M.K.; D’Lima, D.D. Macroscopic and histopathologic analysis of human knee menisci in aging and osteoarthritis. Osteoarthr. Cartil. 2011, 19, 1132-1141. [CrossRef] [PubMed]

55. Pritzker, K.P.; Gay, S.; Jimenez, S.A.; Ostergaard, K.; Pelletier, J.P.; Revell, P.A.; Salter, D.; van den Berg, W.B. Osteoarthritis cartilage histopathology: Grading and staging. Osteoarthr. Cartil. 2006, 14, 13-29. [CrossRef] [PubMed] 
56. Loreto, C.; Lo Castro, E.; Musumeci, G.; Loreto, F.; Rapisarda, G.; Rezzani, R.; Castorina, S.; Leonardi, R.; Rusu, M.C. Aquaporin 1 expression in human temporomandibular disc. Acta Histochem. 2012, 114, 744-748. [CrossRef] [PubMed]

57. Castrogiovanni, P.; Trovato, F.M.; Szychlinska, M.A.; Loreto, C.; Giunta, S.; Scuderi, S.; Passanisi, R.; Fidone, F.; Fagone, P.; Imbesi, R.; et al. Effects of Synthetic Anti-Inflammatory Sterol in CB3V-Induced Myocarditis: A Morphological Study on Heart Muscle Tissue. J. Funct. Morphol. Kinesiol. 2016, 1, 69-89. [CrossRef]

(C) 2016 by the authors; licensee MDPI, Basel, Switzerland. This article is an open access article distributed under the terms and conditions of the Creative Commons by Attribution (CC-BY) license (http://creativecommons.org/licenses/by/4.0/). 